\title{
Temporal analysis of academic performance in higher education before, during and after COVID-19 confinement using artificial intelligence
}

\author{
Laia Subirats ${ }^{1,2}$, Aina Palacios Corral $^{1}$, Sofía Pérez-Ruiz ${ }^{3}$, Santi Fort ${ }^{1}$, Gómez-Moñivas \\ $\operatorname{Sacha}^{3 *}$, \\ 1 Eurecat Academy, Eurecat - Centre Tecnològic de Catalunya, Barcelona, Spain \\ 2 ADaS Lab, Universitat Oberta de Catalunya, Barcelona, Spain \\ 3 Department of Computer Engineering, Universidad Autónoma de Madrid, Madrid, \\ Spain \\ *sacha.gomez@uam.es
}

\begin{abstract}
This study provides the profiles of students and a regression prediction of marks considering data before, during, and after the COVID-19 pandemic. Using a field experiment of 396 students and more than 7400 instances, we have studied the differences in performance considering the temporal distribution of autonomous learning during courses from 2016/2017 to 2020/2021. After applying unsupervised learning, results show 3 profiles of students: continuous students, last-minute students, intense and last-minute students. We have found that the highest success ratio is related to students that work in a continuous basis. However, last minute working is not necessary linked to failure. After applying regression, results show that the mark of the students can be predicted successfully and that the most relevant values are the mean mark in self-evaluation obtained the month before the final exam, the mean mark two months before, the number of attempts two months before and the number of attempts the month before. Results are a little worse but still acceptable if the prediction wants to be made a month before the final exam. This regression is useful to prevent students' wrong learning strategies, and (more effective) to detect malpractices such as copying. We have done all these analysis taking into account the effect of the COVID-19 pandemic, including also a discussion about which factors will be extended in time and which ones are transitory and only due to the confinement.
\end{abstract}

\section{Introduction}

Educational data mining (EDM) has been applied for some years in several initiatives [1] and the use of computers in education is a hot topic nowadays 2]. A great amount of Massive Open Online Courses (MOOCs) has appeared offering their content completely online 3, 3 . (including filmed lectures, problem sets and forums where the community can interact). This change in the learning methodologies (and the massive use of digital information) led to an explosion of data that could be analyzed to improve teaching quality and education (Learning Analytics) [2, 5, 6].

Moreover, between March and April 2020, online learning has been widely used during the COVID-19 confinement [7,8] and the improvement of online learning strategies has become a necessity even to be applied institutionally [9. In this context, 
the term emergency remote learning (ERL) has been coined 10]. Fortunately, due to the interest in distance education in the last years, several e-learning tools were available by that time and widely used [11, including also intelligent tutoring systems (ITS) [12].

In this context, a rigorous analysis of all the elements influencing students' performance in distance learning has become a fundamental requirement to understand the scope of the pandemic in education. This analysis becomes even more relevant in the current situation, where the pandemic is beginning to be left behind and both the transitory influence that occurred during confinement and the effects that will be permanent can be evaluated. In this sense, we will work in the present article with data from students during courses before the pandemic; from the course 2019/2020 (where they were forced to be confined and study at home), and also from the course 2020/2021 where students were not completely confined anymore.

In general, one of the elements that were more affected by the confinement was the time distribution in students' autonomous learning [13]. In the year that they were forced to study at home $(2019 / 2020)$, students worked in a more continuous way due to different factors. On one side, they had more free time since they were not allowed to even go out of their homes. On the other side, more activities were proposed by the teachers to replace the final face-to-face evaluation tests. In the academic year after the confinement (2020/2021), many of these methodologies changed again to the previous format. However, the experience lived by the students and the new habits acquired during confinement could last over time.

The first objective of this article is to analyze the students' profiles and the time distribution in their autonomous learning before, during and after the COVID-19 confinement. The objective is to find out if the effects, beneficial on many occasions, that occurred in confinement are maintained over time. The second objective is to determine if the data collected is adequate to predict students' performance in order to apply measures to correct it if needed. For this objective, we indeed need to know first the different behaviours of students and the correlation to the COVID-19 confinement.

\subsection{Background}

In the past few years, the pandemic has affected our lifestyle and left us in an uncertain time. Many people changed their daily routine, had to stay locked in their house, modified their social life completely and, even today, had to deal with long-lasting or permanent impact from the COVID-19. In terms of educational fields, the students also replaced their presence-based lessons with online courses, had to change their study plan and tried to deal with a completely new educational system. Nowadays, the majority return to a new normality of blended learning or on-site classes.

Some difficulties had to be overcome due to the confinement such as depression, anxiety, poor internet connectivity, and an unfavorable study environment at home. [14 also reports that $70 \%$ of learners were involved, motivated and highlights the necessity of providing resilient education. It has been also reported that the pandemic impacted higher education not only in online classes but also in libraries (closed), social life, personal financial situation, and emotional health [15. However, some studies also pointed out that students' performance during COVID-19 improved [13, 16, 17. In addition, there are some studies such as [18, that collected sociodemographic and health variables, completing a measure of positive change.

Predicting the evolution in students' learning behaviour due to the effects of the past months has become a possibility and may help us to understand the impact of the pandemic. Some studies also tried to understand how some of these factors can modify learners' habits. For example, 19] uses scores to measure the effect of learning changes in students. Detecting any temporal progress of students has been also studied [20] 21] examines the temporal dynamics of self-regulated learning behaviours using the 
multilevel vector autoregression (VAR) model. 22] analyzes the effects of COVID-19 in college students' lessons.

It is clear that this unstable period may have changed students' habits, which can reverberate at their marks. Using temporal data and historical information it is possible to predict the final grade, which can be useful to correct wrong learning strategies. 23 brings analysis articles related to this field. Artificial intelligence has become a tool to predict using historical data, and we can use this tool to try to predict mid and final scores. For example, to predict academic grades and dropout, 24, 25] uses classification, 26, 27] and 28] regression in higher education and high school, respectively. Moreover, students' marks may be predicted using clustering as 29 or applying supervised machine learning algorithms as 30,31 .

Other research inquires that bad attitudes or strategies in arts or soft skills subjects may be a good indicator to predict the future marks of a student [32].

Pandemic changed our lives, and in the future, we may have to deal with similar global situations. Now, we can rely on this data to help our future students in similar circumstances.

\subsection{Purpose}

The aim of this study is to analyze students' performance focusing on time distribution before, during and after the COVID-19 pandemic. We also want to extend the analysis to the possibility of predicting students' performance by unsupervised learning and regression algorithms considering the temporal nature of the data acquired during the last five years. Data has been collected by the e-valUAM web application [33.

These objectives can be achieved by answering the following concrete research questions :

1. Is the temporal representation relevant in the students' profiles?

2. Does the profile of students vary before, during, and after the COVID-19 pandemic?

3. Can wrong learning strategies be predicted?

4. Are the prediction methods effective before, during and after COVID-19 confinement?

\section{Materials and methods}

\subsection{Data gathering}

We used the data of years 2016/2017, 2017/2018, 2018/2019, 2019/2020, and 2020/2021 composing a dataset of 396 students. Participants data was collected in the following manner:

- It does not involve minors.

- It has been collected anonymously. Students have been identified by a numerical code, avoiding gathering any personal information.

- Students have been informed by the lecturers that some information about their activity could be anonymously collected for statistical purposes. The authors of this study did not receive any objections.

- Tasks related to this study did not in any form alter students' activities, classes, or the assessment process. 
The subject of the study was "Applied Computing". This subject was taught through theory lessons and practical classes in the computer laboratory. This course corresponds to 6 ECTS and belongs to the Chemical Engineering Degree in the Faculty of Sciences from Universidad Autónoma de Madrid, Spain. Due to the COVID-19 pandemic, the face-to-face teaching was cancelled on March 11, having a strong impact on the 2019/2020 course since it started in the first week of February and lasted until mid-May. Therefore it should be considered that the first 3 courses were normal ones, 2019/2020 was the year of the pandemic were from 9th March classes were online, and 2020/2021 was the year after COVID-19 confinement with a mixed methodology of face-to-face and virtual teaching.

E-valUAM platform implements computer-adaptive tests (CAT) proposed by Lord in 1980 34 36. CAT allows personalizing the next question of the student considering the student's past answers. In Fig. 1 we show an example of the possible paths that students can face when using e-valUAM. Right answers imply a horizontal movement right on the diagram, while wrong answers imply a vertical drop on the diagram. The lowest grade (grade 0) corresponds to a test where the student has failed all the questions. The highest grade (grade 3) implies that the student has faced questions from all the possible level values $(1,2)$.

Fig 1. Representation of the CAT model. Solid and dotted arrows indicate the path of correct and incorrect answers respectively. Grades are represented at the bottom of the figure, In the $\mathrm{Y}$ axis there is the number of questions and in the circles there is the level of the question.

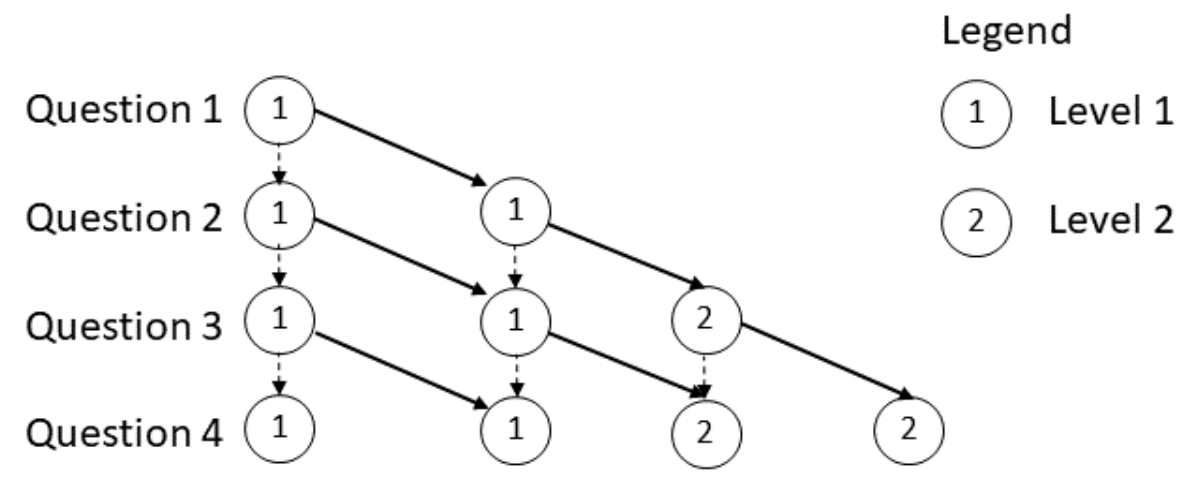

\section{Grade 0 Grade 1 Grade 2 Grade 3}

The performance of this subject is expressed in a 0-10 score. Those Open Answer Test (OA-T) were available from the beginning of the academic year and students used them on a continuous basis. Data from this subject imply results from an adaptive test. This implies that there are some points in the test where students get stacked and some scores are repeated more often than others. In OA-T there is at least one parameter that will change its value with each execution of the application. Questions have the following elements:

- Statement with explicit indication of the modifiable parameter(s).

- Minimum and maximum values of each modifiable parameter.

- Programming code (Matlab in e-valUAM) that calculates the solution to the problem.

- Level of the item. 
Therefore, raw data is composed of two different files or tables. In one there is the e-valUAM data with the id of the student, the timestamp of the answer, and the mark. Indeed, students have several attempts since this test is their main tool in self-evaluation activities. In addition, it should also be taken into account that there are also null attempts in the platform, which are attempts that are not finished and do not have a final mark. The second file is the mark of the final exam, so there are data of the the student's id, the timestamp of the final exam, and the final mark.

For more information about the CAT model and e-valUAM platform refer to the methods section of the article [13].

\subsection{Data cleaning}

The first action before performing the machine learning algorithms has been the cleaning of the data and their transformation in order to include in each instance a student and temporal information. For doing so, a single dataset from the two above-mentioned tables is created with the following attributes:

- month1 [0-10]: the mean mark of the month before the penultimate month

- month1count: number of attempts of the month before the penultimate month

- month2 [0-10]: the mean mark of the penultimate month

- month2count: number of attempts of the penultimate month

- month3 [0-10]: the mean mark of the last month

- month3count: number of attempts of the last month

- mark [0-10]: the final mark of the subject

This data cleaning has been done using the month obtained from the timestamp of the continuous evaluation of each student, and then grouping the information of every student in the same instance across the above-mentioned attributes. Earlier attempts from those mentioned above (before the penultimate month) were not considered because they are not available along all academic years.

Two approaches have been studied: considering null attempts and without considering them. In the first approach, null attempts are considered both in the mean mark of the month and in the count of attempts. In the second approach, null attempts are deleted. Depending on the purpose of the study, one approach or the other one will be used (depending on which one has better result).

All code was implemented in Python 3.8.3, and scikit-learn library 37] was used.

\subsection{Unsupervised learning}

In unsupervised learning, several algorithms can be applied such as k-means, hierarchical clustering and Density-Based Spatial Clustering of Applications with Noise (DBScan). In this work k-means is applied [38] because it offers some advantages as it is relatively simple to implement, scales well, guarantees convergence, easily adapts to new examples and generalizes to clusters of different shapes and sizes, such as elliptical clusters (for more information see [39]). The elbow method is used to find the optimal number of clusters. The method is a heuristic that consists of plotting the explained variation (in this case the within-cluster sum of square (WCSS)) as a function of the number of clusters, and picking the elbow of the curve as the number of clusters to use.

Before applying clustering, Principal Component Analysis (PCA) is performed to reduce dimensionality and the 4 attributes (mean number of months in the 3 months 
before the exam and the final mark) can be represented in a 3-D space. Silhouette score is used to evaluate the clusters [40. The best value if a silhouette score is 1 and the worst value is -1 . Values near 0 indicate overlapping clusters while negative values generally indicate that a sample has been assigned to the wrong cluster because a more similar cluster can be found (for more information see scikit-learn).

\subsection{Regression}

For regression, we have used MLPRegressor with the following parameters for the gridsearchcv:

grid_params_MLPRegressor $=[$

'MLPRegressor__solver': ['lbfgs'],

'MLPRegressor__max_iter': [100,200,300,500],

'MLPRegressor__activation' : ['relu','logistic','tanh'],

'MLPRegressor__hidden_layer_sizes': [(2,), (4,),(2,2),(4,4),(4,2),(10,10),(2,2,2)],]

The StandardScaler was implemented in a Pipeline to standardize the data. This is particularly useful when there are different ranges of numbers in the attributes such as the mean of the mark and the number of attempts. Furthermore, 5-fold cross-validation has been applied.

Several experiments have been performed for regression:

1. Considering $30 \%$ of data randomly to test the regression

2. Not considering the last month data. This is specially useful to be able to warn students in advance.

3. Doing the prediction by years: using $2016 / 2017,2017 / 2018$ data to predict 2018/2019. This implies predicting a normal academic year from two normal academic years.

4. Doing the prediction by years: using $2016 / 2017,2017 / 2018$ data to predict 2019/2020. This implies predicting a confinement / virtual year from two normal academic years.

5. Doing the prediction by years: using $2016 / 2017,2017 / 2018$ data to predict 2020/2021. This implies predicting a mixed / semi-virtual year from two normal academic years.

For evaluating the performance of the regression, the Mean Absolute Error (MAE) has been used because it is one of the used metrics used in the literature (the lower the MAE the better).

\section{Results}

\subsection{Initial statistics of data}

In table 1 we show a summary of the statistics in order to understand the nature of the data of each academic year. We could see that the academic year with the higher mean marks is $2020 / 2021$, followed by $2019 / 2020$. It should be noted that the threshold to pass the subject is 5.5 (instead of the usual 5). 
Table 1. Description of the data. SD stands for standard deviation.

\begin{tabular}{|c|c|c|c|c|}
\hline \multicolumn{5}{|c|}{ Final mark description } \\
\hline & Mean mark & SD mark & \# of fails & \# of students \\
\hline Final evaluation 2016/2017 & 7.79 & 1.51 & 10 & 103 \\
\hline Final evaluation 2017/2018 & 8.23 & 1.35 & 1 & 73 \\
\hline Final evaluation 2018/2019 & 7.63 & 1.40 & 4 & 74 \\
\hline Final evaluation 2019/2020 & 8.57 & 1.09 & 0 & 78 \\
\hline Final evaluation 2020/2021 & 8.60 & 1.02 & 0 & 68 \\
\hline
\end{tabular}

\subsection{Unsupervised learning}

As explained in the methods section, the first step would be to find the best number of clusters. For that, we are going to use the elbow method which is found after computing the Within-Cluster Sum of Square (WCSS) of each cluster from 1 to 9 (see Fig. 2). In Fig. 2 we can see that as the number of clusters increases, the WCSS equals zero. After using the knee locator library and inspecting Fig. 2 visually, we can find that elbow or knee locator is 3 (the best number of clusters is 3 ). After that a representation of the clusters is done in Fig. 2. We can observe that there in colors the 3 clusters (in black, grey and light grey) having as $\mathrm{X}, \mathrm{Y}$, and $\mathrm{Z}$ axis the 3 first components of the PCA. We can see that clusters are clearly differentiated and that black ones are the least abundant cluster, followed by the light grey ones. Finally, the grey one is the cluster with a bigger size.

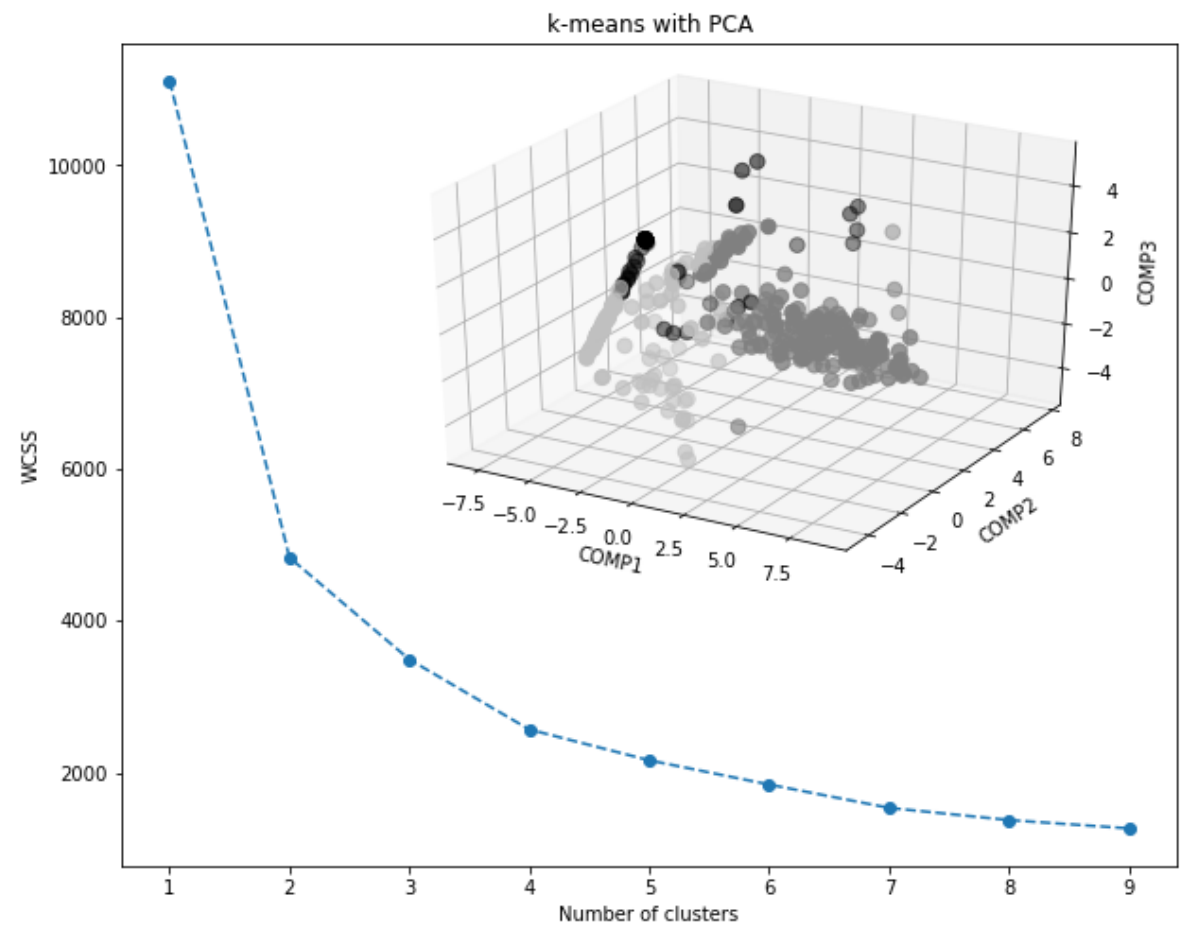

Fig 2. Unsupervised learning learning. Clustering without considering null attempts nor considering the number of attempts. In the outer part of the figure (lower-left) there is the performance according to each number of clusters. In the inner part of the figure (upper-right), the user profiles using unsupervised learning.

In Table 2 we can see that there are three types of profiles of students: the grey ones, 
which are the ones who obtain a high mark and study continuously during the semester, the light grey ones which study hard at the end of the semester; and the black ones, who have a low performance at the continuous evaluation of the semester. It can also be perceived that in the 2019/2020 course there were more students who studied continuously, and that trend decreased in 2020/2021. However, continuous study levels in 2020/2021 have higher levels than before COVID-19 arrived.

Table 2. Description of the three clusters. Mean is used for the dataset attributes for each cluster.

\begin{tabular}{|c|c|c|c|c|}
\hline \multicolumn{5}{|c|}{ Clusters' description } \\
\hline & black & grey & light grey & Total \\
\hline month1 & 0.99 & 4.74 & 0.79 & - \\
\hline month1count & 0.51 & 3.03 & 0.36 & - \\
\hline month2 & 1.0 & 6.9 & 0.64 & - \\
\hline month2count & 0.31 & 3.28 & 0.44 & - \\
\hline month3 & 0.97 & 8.05 & 6.73 & - \\
\hline month3count & 1.38 & 6.59 & 7.45 & - \\
\hline mark & 6.57 & 8.78 & 7.91 & - \\
\hline $2016 / 2017$ & 17 & 17 & 69 & 103 \\
\hline $2017 / 2018$ & 13 & 40 & 20 & 73 \\
\hline $2018 / 2019$ & 20 & 16 & 38 & 74 \\
\hline $2019 / 2020$ & 3 & 74 & 1 & 78 \\
\hline $2020 / 2021$ & 2 & 40 & 26 & 68 \\
\hline size & 55 & 187 & 154 & 396 \\
\hline
\end{tabular}

Finally, regarding the metrics of the cluster, the mean silhouette score is 0.48 and is higher in this experiment than considering null attempts nor the number of attempts. For this reason, the before-mentioned setup was conducted.

\subsection{Regression}

\subsubsection{All courses}

In this experiment, as explained in the methods section, data of all courses of 2016/2017, 2017/2018, 2018/2019, 2019/2020 and 2020/2021 was considered taking $70 \%$ of data for training purposes and the rest $30 \%$ for testing and 5-fold cross-validation.

First, the correlation heatmap of the attributes involved in the study is depicted in Fig. 3. It can be appreciated that there is a high correlation between month1 and month2, month2 and month3, and month3 and mark, and that some correlation values are negative (correlations between month1 and month3count and month2 and month3count).

Finally, the difference between the value and the prediction can be found in Fig. 4 Two thresholds were established: one above 1.5 and above 2 . The values above 1.5 or 2 indicate students that had a better marks than the expected. This could potentially be caused by malpractices such as copying.

Best parameters of the regression prediction have been 'MLPRegressor_-activation': 'relu', 'MLPRegressor_hidden_layer_sizes': $(4,4)$, 'MLPRegressor__max_iter': 100, 'MLPRegressor__solver': 'lbfgs'

\subsubsection{All courses a month before the final exam}

In this experiment, as explained in the methods section, data of all courses of 2016/2017, 2017/2018, 2018/2019, 2019/2020 and 2020/2021 was considered taking $70 \%$ 
Fig 3. Correlation heatmap. Null attempts and the number of attempts are considered.

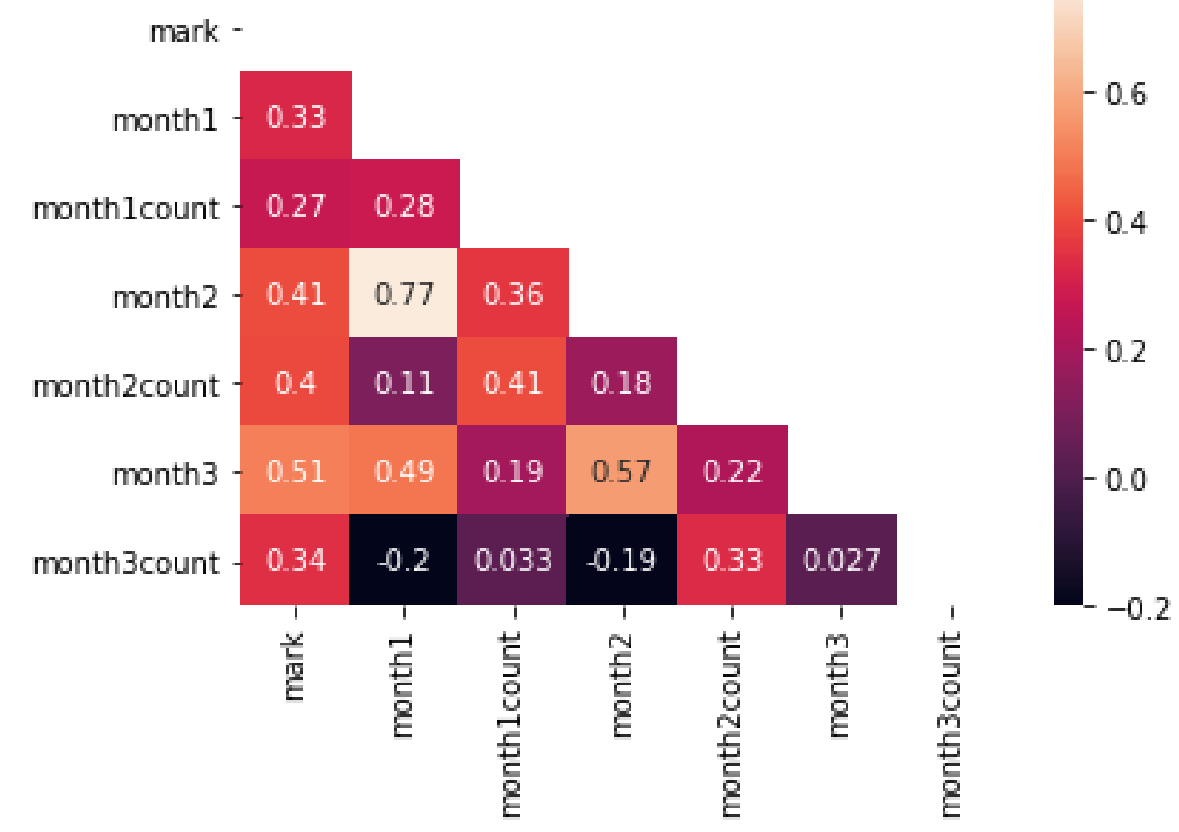

Fig 4. Difference between the value and the prediction. The solid vertical line indicates a threshold from potential learning malpractice. The dotted vertical line indicates the more strict threshold from potential learning malpractice. Null attempts and the number of attempts are considered.

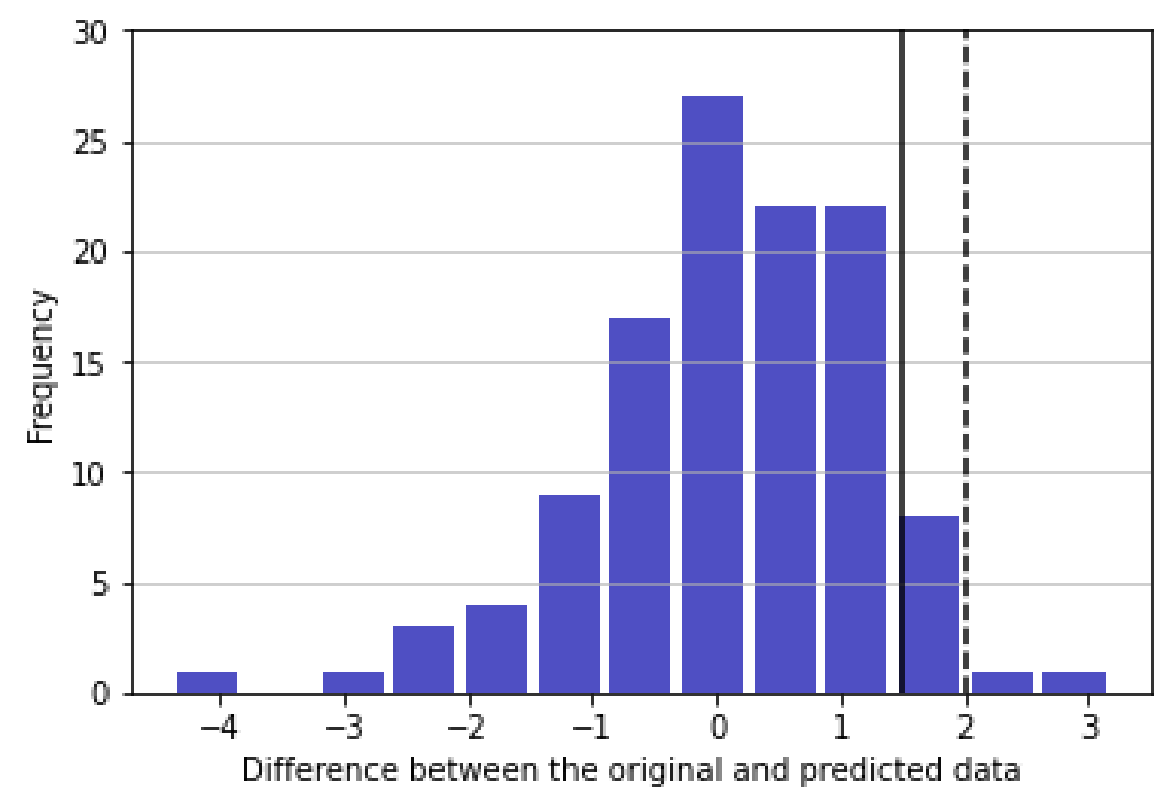

of data for training purposes and the rest 30\% for testing and 5-fold cross-validation. However, in this case, e-valUAM data of a month before the final exam was not 
considered. The purpose of this experiment is to see if the teacher is able to correct students' wrong learning strategies in advance and warn the student.

First, a correlation heatmap of the attributes involved in the prediction of all courses one month before the final exam can be found in Fig. 5. In this case, differently from Fig. 3. month3 and month3count do not appear in the correlation heatmap. It can be appreciated especially that there is a high correlation between month1 and month2, month 2 and mark and month2count and mark, and that in this case there are no negative correlations.

Fig 5. Correlation heatmap one month before the final exam. Null attempts and the number of attempts are considered.

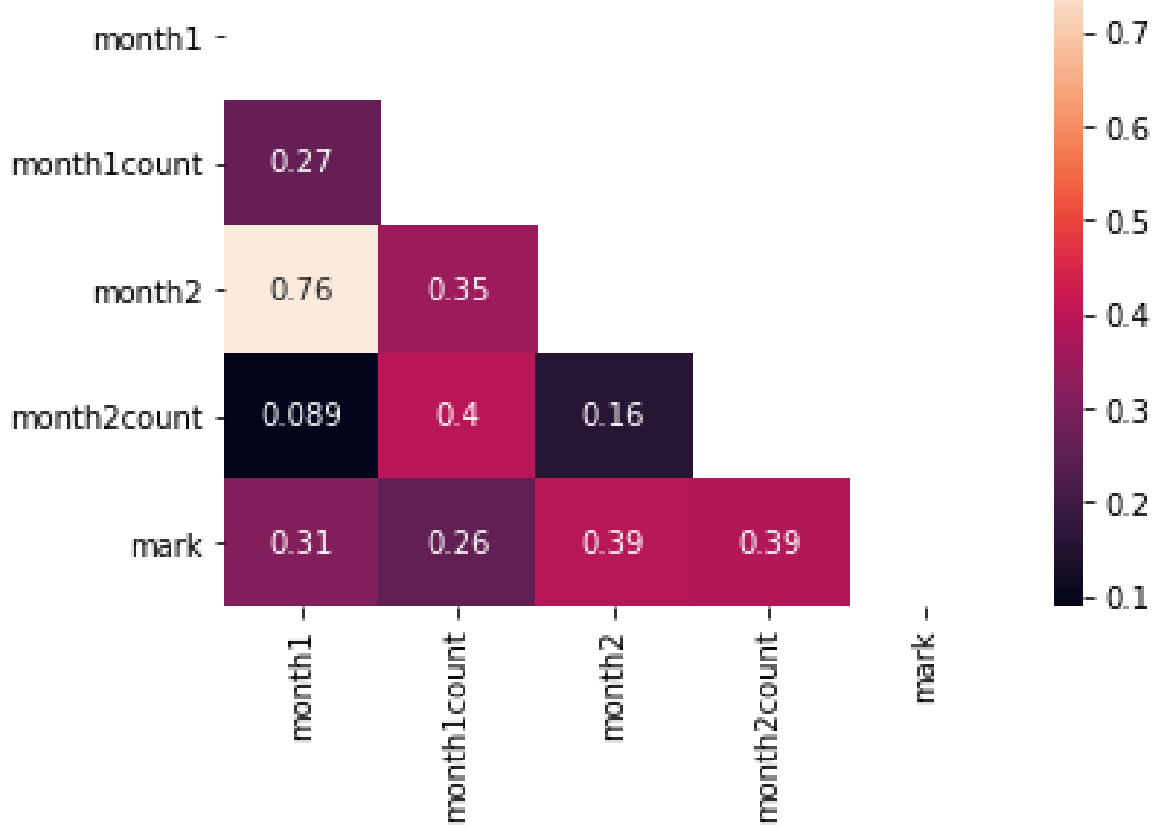

Finally, the difference between the value and the prediction can be found in Fig. 6 . As in Fig. 4 two thresholds were established: one above 1.5 and above 2. The values of the right of the thresholds indicate students that had a better than expected mark. Again, this could potentially be caused by malpractices such as copying. In this case, we can see that potentially the number of potential malpractices is higher than in Fig. 4. This can be caused also because the prediction is not as accurate (it can be noted that the MAE is lower in this case).

In Table 3 there is the detail of students' potential malpractices and wrong learning strategies in both scenarios (with all data and with data at least one month earlier). We can see that the number of potential malpractices is higher when having less data. It should be noted that the threshold to establish potential malpractices is subjective, and depends on the teacher's criteria and available time on reviewing. On the other hand, alerting the students' of their wrong learning strategies could have been useful to students. To establish the first threshold we based on the number of instances related to the marks, the second one es based on the cut-off mark. It should be noted that the mark to pass the subject is 5.5 (instead of 5).

Best parameters of a month before the final exam have been:

'MLPRegressor__activation': 'logistic', 'MLPRegressor__hidden_layer_sizes': (2,), 'MLPRegressor__max_iter': 100, 'MLPRegressor__solver': 'lbfgs' 
Fig 6. Difference one month before the final exam between the value and the prediction. The solid vertical line indicates a threshold from potential learning malpractice. The dotted vertical line indicates the more strict threshold from potential learning malpractice Null attempts and the number of attempts are considered.

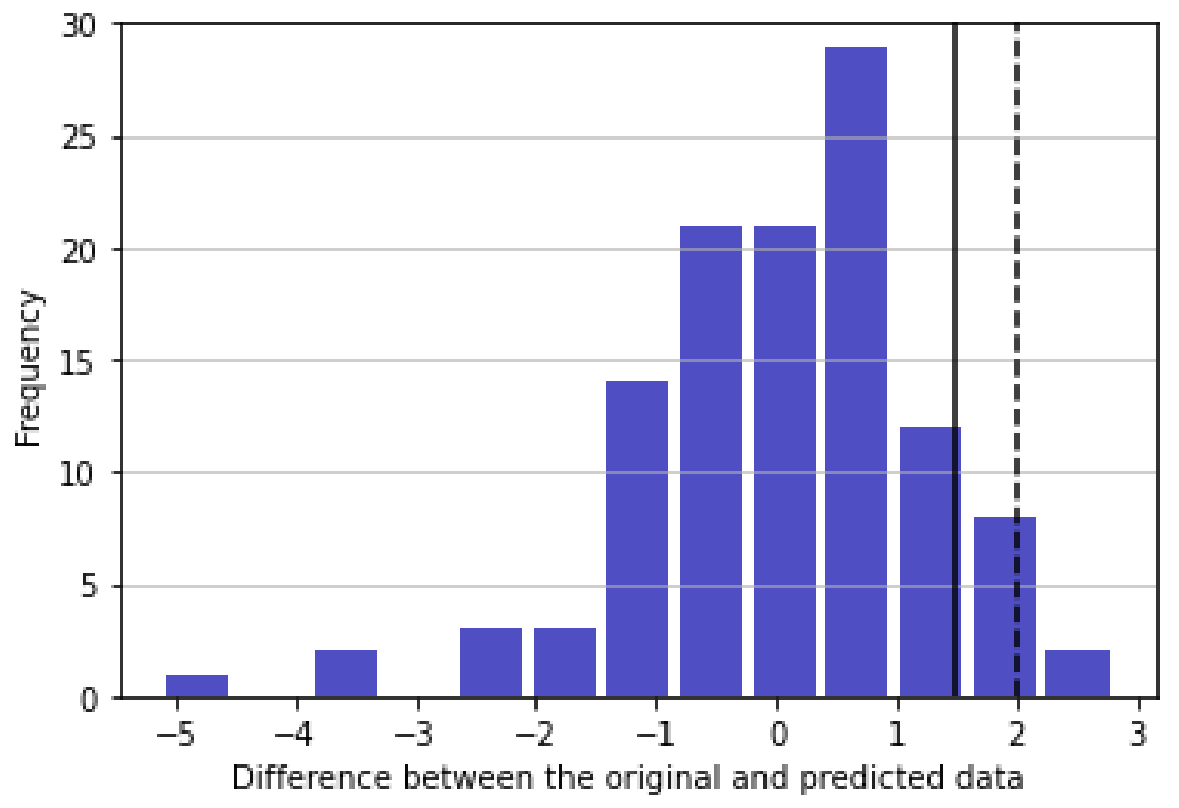

Table 3. Summary of malpractices and wrong learning strategies.

\begin{tabular}{|l|l|l|}
\hline \multicolumn{3}{|c|}{ Malpractices and wrong learning strategies } \\
\hline & All data & Data at least 1 month earlier \\
\hline Malpractices being strict $($ Error $>2)$ & 2 & 3 \\
\hline Malpractices (Error $>1.5)$ & 10 & 11 \\
\hline Stricter wrong learning strategies $($ Mark $<5.5)$ & - & 4 \\
\hline Wrong learning strategies $($ Mark $<7.5)$ & - & 15 \\
\hline
\end{tabular}

\subsubsection{Prediction by years}

In this experiment, as explained in the methods section, three different approaches were followed:

- Prediction of a course before the COVID-19 confinement: 2016/2017, 2017/2018 data to predict 2018/2019

- Prediction of a course during the COVID-19 confinement: 2016/2017, 2017/2018 data to predict 2019/2020

- Prediction of a course after the COVID-19 confinement: 2016/2017, 2017/2018 data to predict $2020 / 2021$

In Fig. 7 a summary of MAE results is shown. We could see that the lowest MAE is the prediction of $2020 / 2021$ (0.80), followed by all courses (0.88), all courses one month before (0.92), prediction of 2018/2019 (0.93), and finally the prediction of 2019/2020 (1.01). 
Fig 7. Summary of MAE results in the experiments.

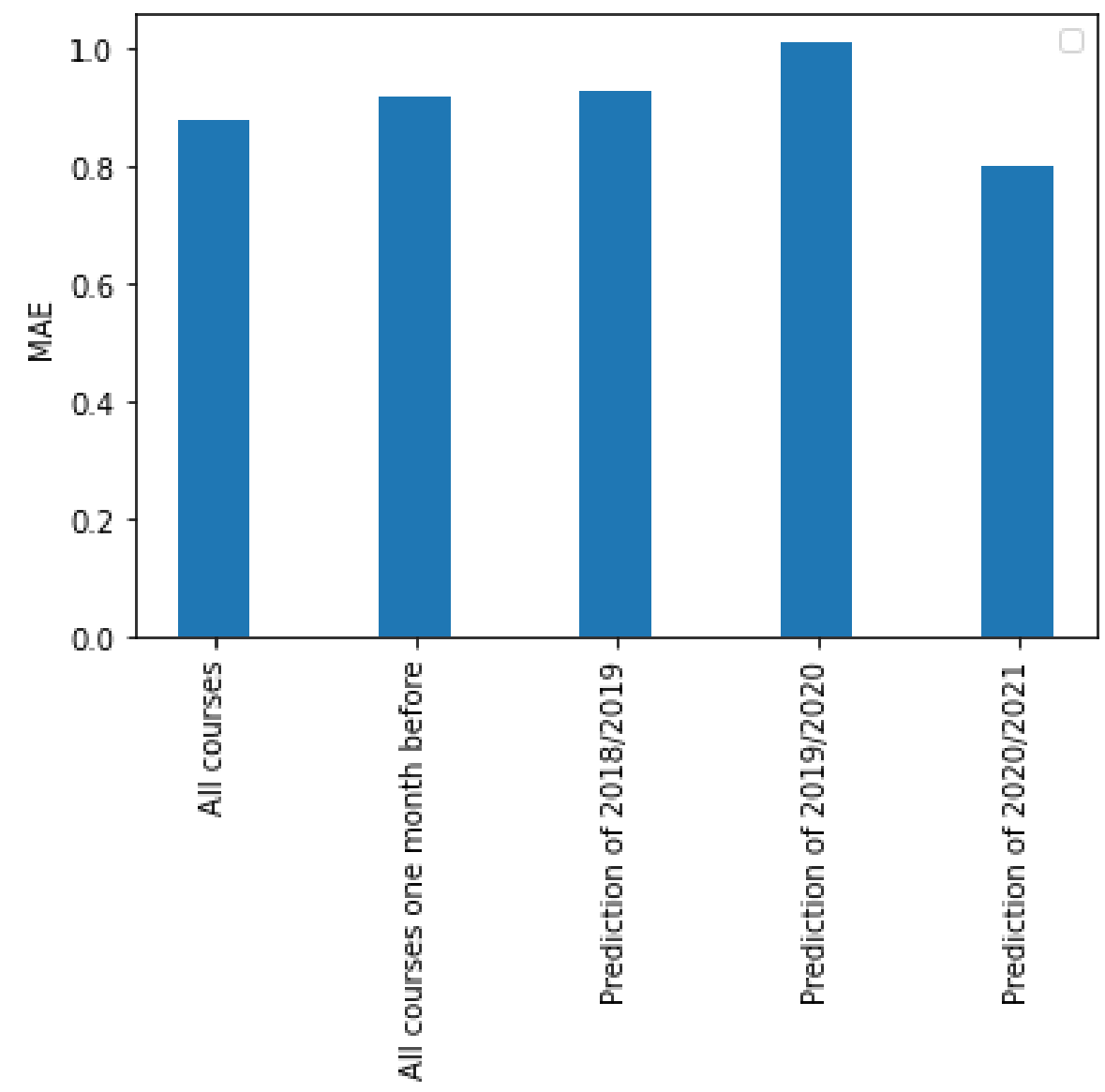

\section{Discussion}

The main objective of this article is to analyze the different learning strategies of students and the influence of the distribution of their autonomous study time. This work is done taking into account the effect of confinement by COVID-19. Additionally, performance prediction strategies are proposed based on the analysis carried out.

In this section the four research questions proposed in the introduction will be answered:

1. Is the temporal representation relevant in the students' profiles?

2. Does the profile of students vary before, during, and after the COVID-19 pandemic?

3. Can wrong learning strategies be predicted?

4. Are the prediction methods effective before, during and after COVID-19 confinement?

Regarding the first research question, we have seen that the temporal representation is relevant because three different types of temporal profiles are clearly obtained. In our analysis, we have observed that there is a group who studies continuously, another one who studies at the end of the term, and the last one with very low performance in the 
continuous evaluation. These results are in agreement with [41], where students who work continuously have a smaller chance of failure. It is worth noting that students who work mainly at the end of the term usually succeeded in their objectives. However, the risk of failure is much bigger for these group. The third group (who was not identified in previous works) does not perform well during the semester and has the highest risk of failure. Results from this point are useful in order to clearly identify the three types of students and to analyze the reasons why different groups have different performance. In that sense, we can distinguish success and failure and two variables. Success is related to group 1 and 2 and failure to group 2 and 3 . The most interesting group is 2 (students who work at the end of the term). In this group, it is very difficult to predict success or failure since both concepts appears often. In terms of data analysis, it is a problem since it is difficult to analyze data removing the last days or weeks because we lose the most important part of the information. In terms of students' perception, it is risky because the chances of success studying only the last few days can send a bad message and be tempting. Students could assume the risk of failure (hoping that they still have some changes of success) and leave most of the hard work to the last few days. It is important for the teachers to motivate students and explain well that it is much better for them to study continuously.

Answering research question2, comparing the different courses of the subject of study, we can see that continuous students are more present in the year 2019/2020, during the COVID-19 confinement. In the following year, this group was also present but with lower number than during the confinement (2020/2021). This information is very interesting since it demonstrates that some good habits lasted one more year at least. The problem now and a good question for future analysis is how long this effect will be present. Another interesting fact is that students' scores were higher in 2020/2021 even when the continuous work started to decrease. There are some facts that could explain this apparent contradiction. First, in course 2020/2021 the restrictions were softer and teachers could use more materials and methodologies with students. Second, students could combine the better conditions with their (still) good habits in continuous working. A further study analyzing the methodologies used in the following years will be essential to confirm these arguments.

Regarding the third research question, in general, it is not easy to predict wrong learning strategies with enough time to apply measures to redirect students' behaviour into a more efficient way of working. We have tried to predict their performance with the whole data set (which includes data until the very last minute) and with data sets that only take into account the information obtained one month before the final exams. In this case, there are some students whom the system predicts are going to pass but in the end, they did not make the exam, which is a source of confusion to the algorithms. This error is difficult to solve because it is present even when we consider a fail all scores under 7.5. This mistakes are mainly due to the lack of information compared to the case when considering all data (it can be noted that the MAE is higher in the last case). In general, students who study until the very last minute are making the exam, so the source of error related to students who quit the subject is not present when considering the whole data set. However, since the predictions including all information are much more accurate, in this case, they could be useful to detect students' wrong learning strategies in a different sense. Regarding students that have a much higher score than expected, (the threshold is established at 1.5 or 2, more strict), we could be detecting students who perhaps could be monitored because they may have potentially copied.

Finally, regarding the last research question, we can confirm that, in the scenario explained in the previous research question, the predictions are still effective in the years when the pandemic hit harder. based in our simulations, we have seen that the best results are for the prediction of 2020/2021 considering 2016/2017 and 2017/2018 
and all results are acceptable. Indeed, our algorithms performance is worse when not including the last month, but this fact happens independently of the academic year (or, at least, there is not a clear correlation between the accuracy and the teaching methodology changes due to the pandemic).

\section{Conclusion}

This study shows the usefulness of determining profiles of students via clustering and regression. The result shows that there are three types of student profiles: the ones who study continuously, the ones who study at the end of the term, and the ones with very low performance in the continuous evaluation. Knowing the type of students is a good help in the learning method since teachers can detect and correct wrong learning strategies and students can use this feedback to try different methodologies when they see that their path is not having a good ending.

Regarding regression, results can successfully predict the final score of the students when considering data from the beginning of the semester to the last day before the final exam. We have seen that the most relevant values are the mean score obtained in their tasks when working autonomously the month before the final exam, the mean two months before, and the number of attempts two months before, and the number of attempts the month before in that order. If the prediction wants to be made a month before the final exam, the error increments but an acceptable prediction is still obtained in some cases. Predicting a month before the final exam is useful to warn students and correct wrong learning strategies. However, in this case, we cannot avoid having some errors, so not all the students at risk can be detected. However, we have seen that our predictions are very helpful to detect malpractices such as copying. It can be highlighted also that predictions are working well before, during and after the confinement due to COVID-19.

For future work, this methodology could be applied to other subjects, universities and countries. It would be particularly interesting to perform a study with students with accessibility problems to the Internet or that have technical limitations in order to tackle Sustainable Development Goal (SDG) 4 - Quality Education (https://www.un.org/sustainabledevelopment/education). The study in different scenarios will be helpful to detect what events are occurring most often in some specific subjects or educational systems. It is worth noting that we have concluded that the different learning strategies applied before and after the confinement, as well as the learning conditions when working at home were very relevant to change students' habits to a more continuous study. This fact could be altered when applying in a different country with different restrictions. Another future direction would be to extrapolate this work to other more subjective subjects such as soft skills (such as arts and language subjects). These subjects can have qualitative information that may enrich the system but may impose a challenge to tackle this type of subjective information. A possible

solution would be to apply sentiment analysis algorithms (there are some libraries that extract the polarity and subjectivity of a sentence such as Textblob) in order to make this subjective information more measurable.

\section{Supporting information}

S1 File. E-valUAM continous evaluation data. Grades of students during continuous evaluation of the subject "Applied Computing".

S2 File. Final marks. Final grade of students of the subject "Applied Computing". 


\section{Acknowledgments}

This study has been partially funded by ACCIÓ, Spain (Pla d'Actuació de Centres Tecnològics 2021) under the project TutorIA. This study was also funded by the Fondo Supera COVID-19 (Project: Development of tools for the assessment in higher education in the COVID-19 confinement).

\section{Author contributions}

Conceptualization: L. Subirats, G.M. Sacha

Formal analysis: L. Subirats, G.M. Sacha

Investigation: L. Subirats, A. Palacios Corral, S. Pérez-Ruiz, G.M. Sacha

Methodology: L. Subirats, A. Palacios Corral, S. Pérez-Ruiz, G.M. Sacha

Project administration: S. Fort, G.M. Sacha

Resources: S. Fort, G.M. Sacha

Software: L. Subirats

Supervision: S. Pérez-Ruiz, S. Fort, G.M. Sacha

Validation: S. Pérez-Ruiz, A. Palacios Corral, S. Fort

Writing - original draft: L. Subirats, A. Palacios Corral, S. Pérez-Ruiz, S. Fort, G.M. Sacha

\section{References}

1. Romero C, Ventura S. Data mining in education. Wiley Interdisciplinary Reviews: Data Mining and Knowledge Discovery. 2013;3(1):12-27.

2. Maldonado-Mahauad J, Pérez-Sanagustín M, Kizilcec RF, Morales N, Munoz-Gama J. Mining theory-based patterns from Big data: Identifying self-regulated learning strategies in Massive Open Online Courses. Computers in Human Behavior. 2018;80:179-196.

3. Rizvi S, Rienties B, Rogaten J, Kizilcec RF. Investigating variation in learning processes in a FutureLearn MOOC. Journal of computing in higher education. 2020;32(1):162-181.

4. McAuley A, Stewart B, Siemens G, Cormier D. The MOOC model for digital practice. Knowledge Synthesis for the Digital Economy. 2010;.

5. Palomo-Duarte M, Dodero JM, Medina-Bulo I, Rodríguez-Posada EJ, Ruiz-Rube I. Assessment of collaborative learning experiences by graphical analysis of wiki contributions. Interactive Learning Environments. 2014;22(4):444-466.

6. Hernández JAC, Alberico AB, Duarte MP, Dellatorre P, Reinoso AJ, Beardo JMD. Teamwork assessment in collaborative projects through process mining techniques. The International journal of engineering education. 2020;36(1):470-482.

7. Motiejūnaite-Schulmeister A, Crosier D. How is Covid-19 affecting schools in Europe?; 2020. https://eacea.ec.europa.eu/national-policies/eurydice/ content/how-covid-19-affecting-schools-europe_en.

8. Studyeu. Impact of COVID-19 on studying abroad in Europe: Overview; 2020. https://www.study.eu/article/ impact-of-covid-19-on-studying-abroad-in-europe-overview. 
9. Aguilera-Hermida AP, Quiroga-Garza A, Gómez-Mendoza S, Villanueva CADR, Alecchi BA, Avci D. Comparison of students' use and acceptance of emergency online learning due to COVID-19 in the USA, Mexico, Peru, and Turkey.

Education and Information Technologies. 2021; p. 1-23.

10. Moser KM, Wei T, Brenner D. Remote teaching during COVID-19: Implications from a national survey of language educators. System. 2021;97:102431.

11. Rodrigues H, Almeida F, Figueiredo V, Lopes SL. Tracking e-learning through published papers: A systematic review. Computers \& Education. 2019;136:87-98.

12. Subirats L, Pérez L, Hernández C, Fort S, Sacha GM. A Granularity-Based Intelligent Tutoring System for Zooarchaeology. Applied Sciences. 2019;9(22):4960.

13. Gonzalez T, De La Rubia M, Hincz KP, Comas-Lopez M, Subirats L, Fort S, et al. Influence of COVID-19 confinement on students' performance in higher education. PloS one. 2020;15(10):e0239490.

14. Kapasia N, Paul P, Roy A, Saha J, Zaveri A, Mallick R, et al. Impact of lockdown on learning status of undergraduate and postgraduate students during COVID-19 pandemic in West Bengal, India. Children and Youth Services Review. 2020;116:105194.

15. Aristovnik A, Keržič D, Ravšelj D, Tomaževič N, Umek L. Impacts of the COVID-19 Pandemic on Life of Higher Education Students: A Global Perspective. Sustainability. 2020;12(20). doi:10.3390/su12208438.

16. Iglesias-Pradas S, Hernández-García Á, Chaparro-Peláez J, Prieto JL. Emergency remote teaching and students' academic performance in higher education during the COVID-19 pandemic: A case study. Computers in Human Behavior. 2021;119:106713.

17. Sanz G, Gil A, Marnet B, Berlanga L, Tordesillas M, Pérez Ruiz S, et al. Students' Performance in French Subjects in the COVID-19 Confinement. In: Eighth International Conference on Technological Ecosystems for Enhancing Multiculturality. TEEM'20. New York, NY, USA: Association for Computing Machinery; 2020. p. 853-858. Available from: https://doi.org/10.1145/3434780.3436690

18. Williams L, Rollins L, Young D, Fleming L, Grealy M, Janssen X, et al. What have we learned about positive changes experienced during COVID-19 lockdown? Evidence of the social patterning of change. PLoS One. 2021;16(1):e0244873.

19. N - Kim K. The Effect of the Math Emporium Instructional Method on Students' Performance in College Algebra. European Journal of Science and Mathematics Education. 2017;5(1):1-13.

20. Kinnebrew JS, Segedy JR, Biswas G. Analyzing the temporal evolution of students' behaviors in open-ended learning environments. Metacognition and Learning. 2014;9(2):187-215. doi:10.1007/s11409-014-9112-4.

21. Li S, Du H, Xing W, Zheng J, Chen G, Xie C. Examining temporal dynamics of self-regulated learning behaviors in STEM learning: A network approach. Computers \& Education. 2020;158:103987. doi:10.1016/j.compedu.2020.103987. 
22. Ilieva G, Yankova T, Klisarova-Belcheva S, Ivanova S. Effects of COVID-19

Pandemic on University Students' Learning. Information. 2021;12(4):163.

doi:10.3390/info12040163.

23. Hellas A, Ihantola P, Petersen A, Ajanovski VV, Gutica M, Hynninen T, et al. Predicting academic performance: a systematic literature review. In: 23rd annual ACM conference on innovation and technology in computer science education; 2018. p. pp. $175-199$.

24. Rovira S, Puertas E, Igual L. Data-driven system to predict academic grades and dropout. PLoS one. 2017;12(2):e0171207.

25. Tsiakmaki M, Kostopoulos G, Koutsonikos G, Pierrakeas C, Kotsiantis S, Ragos O. Predicting University Students' Grades Based on Previous Academic Achievements. In: 2018 9th International Conference on Information, Intelligence, Systems and Applications (IISA). IEEE; 2018. p. 1-6.

26. Karlos S, Kostopoulos G, Kotsiantis S. Predicting and interpreting students' grades in distance higher education through a semi-regression method. Applied Sciences. 2020;10(23):8413.

27. Sravani B, Bala MM. Prediction of student performance using linear regression. In: 2020 International Conference for Emerging Technology (INCET). IEEE; 2020. p. $1-5$.

28. Márquez-Vera C, Cano A, Romero C, Noaman AYM, Mousa Fardoun H, Ventura S. Early dropout prediction using data mining: a case study with high school students. Expert Systems. 2016;33(1):107-124.

29. Lopez MI, Luna JM, Romero C, Ventura S. Classification via clustering for predicting final marks based on student participation in forums. International Educational Data Mining Society. 2012;.

30. Subirats L, Fort S, de Haro N, Sacha G. Data Analysis for the prediction and correction of students' wrong learning strategies. In: Proceedings of the Seventh International Conference on Technological Ecosystems for Enhancing Multiculturality; 2019. p. 260-264.

31. Subirats L, Fort S, Atrio S, Sacha GM. Artificial Intelligence to Counterweight the Effect of COVID-19 on Learning in a Sustainable Environment. Applied Sciences. 2021;11(21):9923.

32. Haro-Garcia Nd, Comas-Lopez M, Hincz KP, Mazalu M, Sacha G. Soft Skills assessment in Art and Globalization. In: Proceedings of the Sixth International Conference on Technological Ecosystems for Enhancing Multiculturality; 2018. p. 199-204.

33. Molins-Ruano P, González-Sacristán C, Díez F, Rodriguez P, Sacha GM. An Adaptive Model for Computer-Assisted Assessment in Programming Skills. International Journal of Engineering Education. 2015;31:764.

34. Hambleton RK, Swaminathan H, Rogers HJ. Fundamentals of item response theory. vol. 2. Sage; 1991.

35. Lord FM. Applications of item response theory to practical testing problems. Lawrence Erlbaum Associates, Hillsdale, NJ; 1980.

36. Wainer H. Computerized adaptive testing: a primer. Hillsdale, NJ: LEA; 1990. 
37. Scikit-learnCommunity. Scikit-learn, Machine Learning in Python; 2021. https://scikit-learn.org

38. Arthur D, Vassilvitskii S. k-means++: The advantages of careful seeding. Stanford; 2006.

39. GoogleDevelopers. k-Means Advantages and Disadvantages; 2021. https://developers.google.com/machine-learning/clustering/ algorithm/advantages-disadvantages.

40. Rousseeuw PJ. Silhouettes: a graphical aid to the interpretation and validation of cluster analysis. Journal of computational and applied mathematics. 1987;20:53-65.

41. de la Rubia MA, Sacha GM. Adaptive Tests as a Tool for Evaluating Work Groups in Engineering. International Journal of Engineering Education. 2020;36(411). 